\title{
Revisión de las lesiones en el tenis júnior y universitario
}

\author{
Oriol Amer Orfila y Josep Campos-Rius
}

\section{RESUMEN}

En este trabajo se realiza una revisión sobre el estado actual de las lesiones en el tenis adolescente con el fin de determinar cuáles son las lesiones más frecuentes. Se analizan diversos artículos de revisión y de intervención obteniendo que el tobillo, el hombro, la espalda y la rodilla, son las lesiones más comunes en el tenis júnior y universitario.

\author{
Palabras clave: incidencia \\ lesiva, tipología lesiva, tenis \\ adolescente
}

Recibido: 17 Enero 2019

Aceptado: 20 Enero 2019

Autor correspondiente: Oriol Amer Orfila.

Correo electrónico: oriolamerorfila@gmail.com y josepcampos@gmail.com

\section{INTRODUCCIÓN}

El tenis es uno de los deportes más populares en todo el mundo que atrae a personas de diferentes grupos de edades y con una participación mayor a los 200 países afiliados a la "Federación Internacional de Tenis" (ITF, 2017). En los últimos años, la evolución del tenis caracterizado por el aumento de la fuerza y velocidad del juego, ha provocado la aparición de nuevas patologías, y en muchos casos en edades muy tempranas antes no detectadas en estas categorías (Clínica MAPFRE de Medicina del Tenis \& Fundación MAPFRE, 2015). Por estas razones, un buen conocimiento del estado actual de las lesiones en el tenis en las categorías juveniles hasta la etapa universitaria es necesario que se tenga presente para entrenadores y preparadores físicos.

El objetivo de este trabajo es analizar la bibliografía científica existente sobre las lesiones en jugadores júniors y universitarios de tenis, determinando cuáles son las lesiones más frecuentes.

\section{MËTODO}

En el presente trabajo se ha llevado a cabo una revisión de la literatura existente sobre las lesiones en jugadores adolescentes de tenis (10-22 años). En este trabajo se han incluido tanto artículos experimentales de investigación científica como otros artículos de revisión sistemática sobre la temática establecida. Los apartados que se han incluido en el artículo de los cuales se han analizado sus resultados, han sido: incidencia lesiva, tipología de las lesiones y localización de las lesiones por región anatómica.

\section{INCIDENCIA LESIVA}

Actualmente, las estadísticas que se tienen sobre la incidencia lesiva en tenistas de categorías júniors, son muy dispares debido a dos factores principalmente. Muchos de los estudios estadísticos de recogida de datos a nivel júnior datan desde los años 1989 hasta 2015 (Lanese et al, 1990; Weijermans et al, 1998; Spinks et al., 2006; Hjelm et al., 2010; Colberg et al., 2015; Pluim et al., 2016) obteniendo una variabilidad de resultados muy grande debido a la generalidad de la búsqueda en los primeros años y la especificidad de ésta en las últimas investigaciones.

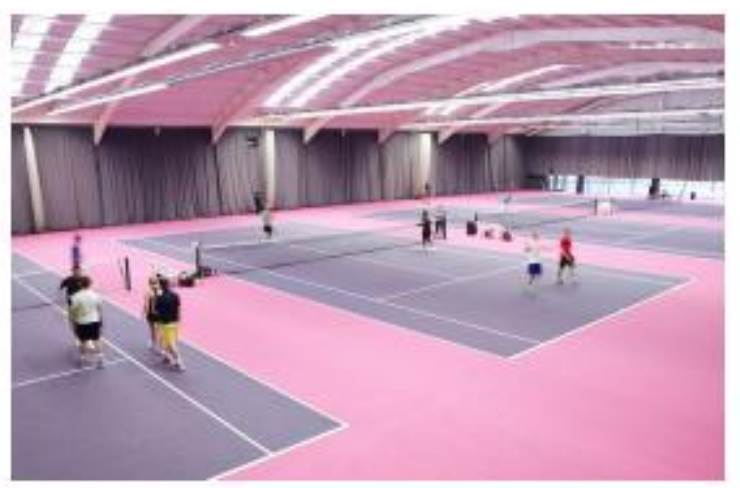


En segundo lugar, no existe un criterio común entre los autores para determinar qué se considera por lesión y por tanto los criterios de inclusión de lesiones son dispares entre los estudios tal y como podemos observar en la figura 1.

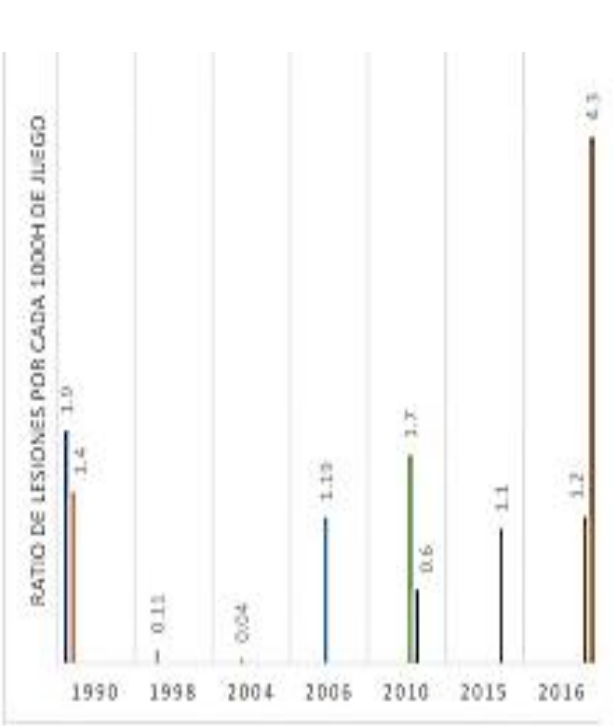

- Hombres flatece ab. 19:01

- Mujeres |Lanese et al, 2990)

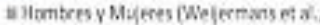

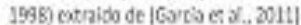

n Hombres y Muictes (US Study estraide de phi.in of al 2086

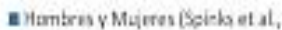
$2006 \%$

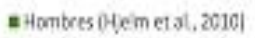

- Mujeres |Hijetmet al, 2010)

E Hemiers y Mujeres. Lesienes ag-das (Coberg et 31.2015)

E Hombres y Mujeres. Lesiones sgodas (himimat al, 2016)

n Hombres y Mtictes. Lesienes por vatre sun |Pum et at, 2010)

Figura 1. Resultadas de la incidencia lesha por cada 1000 h de juego en el tenis júnior y universitaria. Fuente: elaboroción propia.

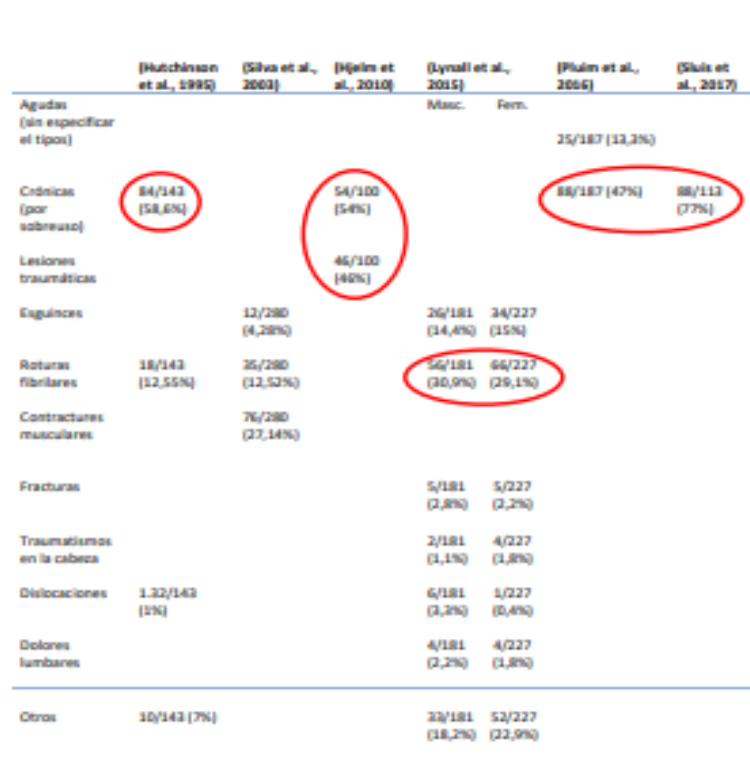

Tabla 1: Resultodos tipologia de las lesiones en el tenis júnior y universitorio entre los oños 1995 y 2017 . Fuente: elaboración propia.

\begin{tabular}{|c|c|c|c|c|c|c|c|c|c|}
\hline & 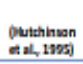 & O(teln of al, 2010) & 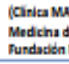 & 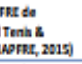 & $\begin{array}{l}\text { (Cobarg at } \\
\text { LL, 2015) }\end{array}$ & alanal et & a al, 2015) & $\begin{array}{l}\text { (Plimm } \\
\text { 2014) }\end{array}$ & et al, \\
\hline & & Male Ternile & Deginnen & $\begin{array}{l}\text { Hith } \\
\text { competition }\end{array}$ & & Mala & Tenat & $\begin{array}{l}\text { Asute } \\
\text { Injury }\end{array}$ & $\begin{array}{l}\text { Chenic } \\
\text { hury }\end{array}$ \\
\hline $\begin{array}{l}\text { Tataloy } \\
\text { paie }\end{array}$ & & $\begin{array}{l}1 / 27 \\
(12 x)\end{array}$ & $17, \operatorname{Aox}$ & $42,0 \mathrm{x}$ & $7 / 19$ (115) & $\begin{array}{l}22 / 1 \mathrm{~m} \\
(127,7 \mathrm{x})\end{array}$ & $\begin{array}{l}47 / 227 \\
{[20,151]}\end{array}$ & $\begin{array}{l}9 / 28 \\
(3 \mathrm{ES})\end{array}$ & $\begin{array}{l}11 / \mathrm{m} \\
{[12,5 \mathrm{~S} \mid}\end{array}$ \\
\hline $\begin{array}{l}\text { Caderay } \\
\text { ingyo }\end{array}$ & $\begin{array}{l}28 / 413 \\
(18 X)\end{array}$ & & seas & $15,50 x$ & $1 / 190(1 \mathrm{X})$ & $\begin{array}{l}16 / 1 \mathrm{in} \\
{[\mathrm{A} / \mathrm{N}]}\end{array}$ & $\begin{array}{l}15 / 227 \\
|\alpha, E x\rangle\end{array}$ & $\begin{array}{l}5 / 25 \\
(25 \mathrm{~N})\end{array}$ & $\begin{array}{l}4 \mathrm{in} \\
(4,5 \mathrm{~N})\end{array}$ \\
\hline Modtha & $1 / 43(2 \times)$ & 1x) & $2,40 x$ & $2450 \mathrm{~s}$ & $5 / 23(112)$ & $\begin{array}{l}1 \mathrm{a} / 1 \mathrm{in} \\
0, \mathrm{~N}]\end{array}$ & $\begin{array}{l}11 / 227 \\
(8,7 \times)\end{array}$ & $\begin{array}{l}1 / 25 \\
(125)\end{array}$ & 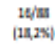 \\
\hline $\begin{array}{l}\text { Muhecay } \\
\text { maro }\end{array}$ & 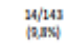 & & $10,0 \mathrm{x}$ & אסק, אנ1, & $1 / 39(2 \mathrm{X})$ & 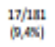 & $\begin{array}{l}21 / 227 \\
|\alpha, 2 x|\end{array}$ & $\begin{array}{l}2 / 25 \\
\mid[\mathrm{m} \mid\end{array}$ & (in) \\
\hline Mantro & $12 / 13]|x|$ & & $15,10 \mathrm{x}$ & 20,100x & $7 / 13 \mid \mathrm{ax})$ & $\begin{array}{l}20 / 11 \mathrm{nn} \\
(21,4 \mathrm{~K})\end{array}$ & $\begin{array}{c}27 / 227 \\
(12 \mathrm{x})\end{array}$ & & $\begin{array}{l}1 / \mathrm{m} \\
\text { (aEs) }\end{array}$ \\
\hline tupuda & $\begin{array}{l}17 / 414 \\
(12 x)\end{array}$ & & $2,0 \times$ & $14,20 \mathrm{x}$ & $\begin{array}{c}8 / 318 \\
(25,45)\end{array}$ & $\begin{array}{l}\mathrm{sa} / \mathrm{in} \\
(\mathrm{MB}, \mathrm{OK})\end{array}$ & $\begin{array}{l}48 / 217 \\
{[177,65]}\end{array}$ & & $\begin{array}{l}1 \mathrm{1} / \mathrm{m} / \mathrm{m} \\
(27 \mathrm{~s})\end{array}$ \\
\hline codo & 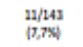 & & $7, \mathrm{Jax}$ & 225 & $2 / 10$ (SK) & $\begin{array}{l}17 / 111 \\
0,250]\end{array}$ & $\begin{array}{l}11 / 227 \\
\beta, 7 \times)\end{array}$ & & j/min \\
\hline Derra & $1 / 1 / 11(2 \times)$ & & & & & & & & 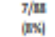 \\
\hline Munb & $\begin{array}{l}27 / 41 / 43 \\
\text { (1\%) }\end{array}$ & & & & $3 / 190(\mathbf{I X})$ & $\begin{array}{l}11 / 1112 \\
0,150]\end{array}$ & $\begin{array}{c}12 / 217 \\
\text { [15] }\end{array}$ & & \\
\hline $\begin{array}{l}\text { Parte inferiar } \\
\text { pierna (no } \\
\text { elpeatitcasa) }\end{array}$ & & & & & & $\begin{array}{l}12 / 1 \mathrm{~m} \\
{[\mathrm{a}, \mathrm{Ex})}\end{array}$ & $\begin{array}{l}2121227 \\
{[1115 \mathrm{~s}]}\end{array}$ & & \\
\hline $\begin{array}{l}\text { Antetrautn } \\
\text { Abdeminaln } \\
\text { No identhicado }\end{array}$ & $4 / 441(\pi x)$ & & & & $\begin{array}{l}1 / 190(1 \mathrm{~N}) \\
1 / 190(1 \mathrm{X}) \\
1 / 190(1 \mathrm{~N})\end{array}$ & & & & \\
\hline Otron & & & & & & $\begin{array}{l}1 / 141 \\
(1, \pi) \mid\end{array}$ & (1,127) & $\begin{array}{l}4 / 25 \\
\mid 18 x)\end{array}$ & $\begin{array}{l}\text { san } \\
(\operatorname{san} x)\end{array}$ \\
\hline
\end{tabular}

Tabla 2: Resultados localización de las lesiones por región anatómica en el tenis júnior y universitario entre los años 1995 y 2016. Fuente: elaboración

\section{TIPOLOGÍA DE LAS LESIONES}

Las diferencias obtenidas entre los tipos de lesiones analizadas pueden venir dadas por varios factores como: el año en el que se realizó el estudio, las características físicas de los sujetos analizados, las diferentes superficies de pista en la que se 
desarrolla el estudio, y el criterio de registro de los datos según cada uno de los autores.

En la Tabla 1 se exponen los datos extraídos de cada uno de los artículos de las que se ha realizado el análisis estadístico y de los cuales se han obtenido los siguientes resultados:

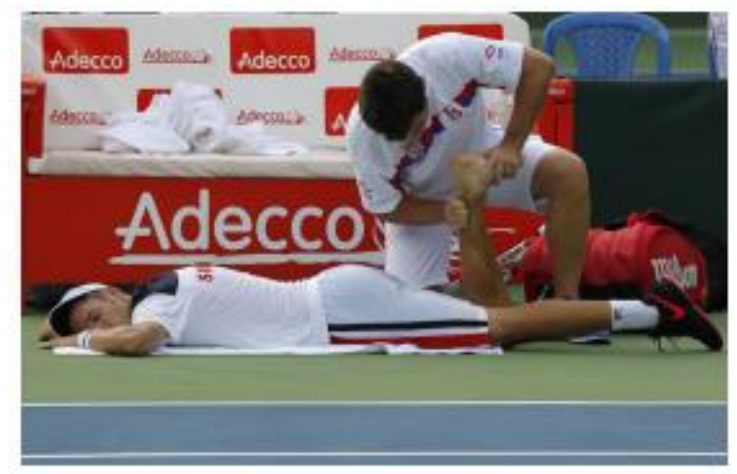

- Las lesiones agudas representan el 20,5\% mientras que las crónicas el $59,15 \%$ y el $20 \%$ restante aproximado son lesiones no especificadas o de menor interés.

- Dentro de las lesiones agudas, podemos afirmar que, las más frecuentes en el tenis son las de carácter muscular como sobrecargas, distensiones y especialmente las roturas fibrilares $(12,52$ a $30,9 \%)$ seguidos de los esguinces $(4,28-17 \%)$.

- Las fracturas (2-2,8\%), dislocaciones $(0,4-3,3 \%)$ y traumatismos (1,1 a 1,8\%) son muy poco frecuentes en este deporte.

\section{LOCALIZACIÓN DE LAS LESIONES POR REGIÓN ANATÓMICA}

En los últimos años se han publicado varios estudios en jugadores júniors y universitarios de tenis (Hutchinson et al., 1995; Silva et al., 2003; Hjelm et al., 2010; Lynal et al., 2015; MAPFRE et al., 2015; Colberg et al., 2015; Pluim et al., 2016; Sluis et al., 2017) donde se han registrado las lesiones por región anatómica. En la tabla 2 podemos ver un resumen con los datos registrados entre los años 1989 y 2016, de los cuales se han obtenido los siguientes resultados:

- La extremidad inferior es la que presenta un mayor rango lesivo (2-42,7\%), seguida de la extremidad superior $(1,1 \mathrm{a}$ $33,7 \%)$ y en último lugar el CORE (3-17 6\% )

- Las zonas en las que podemos encontrar valores más altos de incidencia lesiva son el tobillo $(21,6 \pm 10,04 \%)$, el hombro $(16,2 \pm 6,97 \%)$, la espalda $(13,8 \pm 4,84 \%)$ y la rodilla $(12,7 \pm 7,55 \%)$.

En esta revisión se demuestra que:
- No podemos establecer una tendencia en la incidencia lesiva de los ratios lesivos en la evolución de los años.

- Las lesiones crónicas $(59,15 \%)$ son más frecuentes que las agudas (20,5\%). Dentro las lesiones agudas, las más comunes son las roturas fibrilares $(12,52$ a $30,9 \%)$ y en segundo lugar los esguinces (4,28-17\%). Las fracturas, contusiones y traumatismos son muy poco frecuentes debido a la naturaleza del juego.

- La extremidad inferior es la que presenta un mayor rango lesivo (2-42,7\%), seguida de la extremidad superior $(1,1$ a $33,7 \%)$ y en último lugar el CORE (3-17,6\%). El tobillo $(21,6$ $\pm 10,04 \%)$ del hombro $(16,2 \pm 6,97 \%)$, la espalda $(13,8 \pm$ $4,84 \%)$ y la rodilla $(12,7 \pm 7,55 \%)$ son las regiones que presentan valores más altos de incidencia lesiva en la muestra analizada.

\section{REFERENCIAS}

Clínica MAPFRE de Medicin del Tenis \& Fundación MAPFRE. (2015). Las lesiones en el tenis y su prevención, 1-20. Recogido de https://www.fundacionmapfre.org/documentacion/ publico/i18n/catalogo_imagenes/grupo.cmd?path $=1087681$

Colberg, R., Aune, K., Choi, A. \& Fleisig, G. (2015). Incidence and Prevalence of Musculoskeletal Conditions in Collegiate Tennis Athletes. Medicine \& Science in Tennis, 20(3).

Hjelm, N., Werner, S. \& Renstrom, P. (2010). Injury profile in junior tennis players: a prospective two-year study. Knee surgery, sports traumatology, arthroscopy, 18(6), 845-850. https://doi.org/10.1007/s00167-010-1094-4

Hutchinson, M., Laprade, R., Burnett, Q., Moss, R. \& Terpstra, J. (1995) Injury surveillance at the USTA Boys' Tennis Championships: a 6yr study. Medicine and science in sports and exercise, 27(6), 826831. https://doi.org/10.1249/00005768-199506000-00006

International Tennis Federation (2017). National Association Updates. Recogido de http://www.itftennis.com/about/misc/ nationalassociation-updates.aspx

Lanese, R., Strauss, R., Leizman, D. \& Rotondi, A. (1990). Injurymand disability in matched men's and women's intercollegiate sports. American Journal of Public Health, 80(12), 1459- 1462. https://doi.org/10.2105/AJPH.80.12.1459

Lynall, R. C., Kerr, Z. Y., Djoko, A., Pluim, B., Hainline, B. \& Dompier, T. P. (2015). Epidemiology of National Collegiate Athletic Association men's and women's tennis injuries, 2009/2010- 2014/2015. ritish Journal Sports Medicine https://doi.org/10.1136/bjsports-2015$\underline{095360}$

Pluim, B., Loeffen, F., Clarsen, B., Bahr, R. \& Verhagen, E. (2016). A oneseason prospective study of injuries and illness in elite junior tennis. Scandinavian journal of medicine \& science in sports, 26(5), 564-571. https://doi.org/10.1111/sms.12471

Silva, R., Takahashi, R., Berra, B., Cohen, M. \& Matsumoto, M. (2003) Medical assistance at the Brazilian juniors tennis circuit-a oneyear prospective study. Journal of science and medicine in sport, 6(1), 14-18. https://doi.org/10.1016/S1440-2440(03)80004-X 
Sluis, A., Brink, M., Pluim, B. Verhagen, E. Elferink-Gemser, M. \& Visscher, C. (2017). Is risk-taking in talented junior tennis players related to overuse injuries?. Scandinavian journal of medicine \& science in sports, 27(11), 1347-1355. https://doi.org/10.1111/sms.12729

Spinks, A., Macpherson, A., Bain, C. \& McClure, R. (2006). Injury risk from popular childhood physical activities: results from an Australian primary school cohort. Injury Prevention, 12(6), 390-394. https://doi.org/10.1136/ip.2006.011502

Weijermans, D. \& Van Mechelen, W. (1998). Blessures bij.

CONTENIDO ITF ACADEMY RECOMENDADO (HAZ CLICK ABAJO)

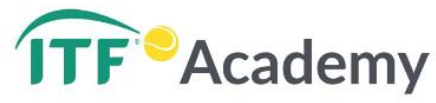

Derechos de Autor (c) 2019 Oriol Amer Orfila y Josep Campos-Rius

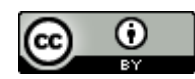

Este texto está protegido por una licencia CreativeCommons 4.0 .

\begin{abstract}
Usted es libre para Compartir — copiar y redistribuir el material en cualquier medio o formato- y Adaptar el documento - remezclar, transformar y crear a partir del material- para cualquier propósito, , incluso para fines comerciales, siempre que cumpla la condición de:

Atribución: Usted debe dar crédito a la obra original de manera adecuada, proporcionar un enlace a la licencia, e indicar si se han realizado cambios. Puede hacerlo en cualquier forma razonable, pero no de forma tal que sugiera que tiene el apoyo del licenciante o lo recibe por el uso que hace de la obra.

Resumendelicencia - Textocompletodelalicencia
\end{abstract}

